\title{
Cumplimiento de una estrategia de higiene de las manos en ambientes asistenciales*
}

\author{
Compliance with a hand hygiene strategy in healthcare settings \\ Observância da estratégia de higiene das mãos em ambientes assistenciais
}

\author{
Mercedes Salcedo-Cifuentes ${ }^{\text {a }}$ \\ Universidad del Valle, Colombia \\ mercysal2003@yahoo.com \\ ORCID: https://orcid.org/0000-0002-9681-6893 \\ Cecilia Andrea Ordónez-Hernández \\ Universidad Libre, Colombia \\ ORCID: https://orcid.org/0000-0001-9296-3768 \\ Andrea Patricia Calvo-Soto
Universidad Santiago de Cali, Colombia, Colombia
ORCID: https://orcid.org/0000-0002-1723-9021
}

DOI: https://doi.org/10.11144/Javeriana.ie22.cehm

Recibido: 21 Diciembre 2018

Aceptado: 12 Diciembre 2019

Publicado: 22 Julio 2020

\section{Resumen:}

Introducción: Si bien la higiene de las manos es la medida más importante en la mitigación del riesgo biológico en ambientes hospitalarios, evidencia científica muestra un escaso cumplimiento de las recomendaciones entre los trabajadores asistenciales. Objetivo: Evaluar el cumplimiento de cinco dimensiones que fortalecen la estrategia de higiene de las manos para la prevención del riesgo biológico en ambientes asistenciales. Método: Estudio observacional, de corte transversal, en cinco instituciones prestadoras de servicios de salud. Se aplicó estadística descriptiva. Con el Test de Medianas se evaluó si había diferencias signicativas entre el grupo de profesionales por institución de salud, considerando significante una $p \leq 0,05$. El análisis finalizó con un análisis discriminante. Resultados: La representatividad estuvo en las instituciones hospitalarias de primer nivel en las cuales el personal de enfermería, seguido por los médicos, tuvo un mejor cumplimiento del protocolo de lavado de manos. Este porcentaje se vio impactado por el menor cumplimiento de suministro de insumos e infraestructura. Hay una perfecta diferenciación entre los resultados del municipio de Cali con relación al de Caicedonia, en contraste con los de Popayán, Buga y Buenaventura. Conclusión: Se encontró un incumplimiento del protocolo de higiene de las manos, con una calificación inaceptable para los factores de insumos/infraestructura y técnica de higiene de las manos, respectivamente. Palabras clave: desinfección de las manos, servicios preventivos de salud, higiene de las manos, prevención de enfermedades.

\begin{abstract}
:
Introduction: Although hand hygiene is the most important measure for biological risk mitigation in hospital settings, there is scientific evidence of low compliance among healthcare workers with the established recommendations. Objective: To assess compliance with five dimensions that strengthen the hand hygiene strategy for biological risk prevention in healthcare settings. Method: Observational, cross-sectional study in five institutions providing healthcare services with the application of descriptive statistics. The Median Test evaluated whether there were significant differences among groups of professionals per health institution, considering a $\mathrm{p} \leq 0.05$ to be significant. The analysis was completed with a discriminant analysis. Results: First-level hospital institutions showed representative results where the nursing staff, followed by physicians, had a better compliance with the handwashing protocol. This percentage was impacted by the lower compliance with the provisioning of supplies and infrastructure. There is a perfect differentiation between the results of the municipality of Cali compared those of Caicedonia, and in contrast with the results of Popayán, Buga, and Buenaventura. Conclusion: Compliance with the hand hygiene protocol was seen, with an assessment rated as unacceptable for the factors related to supplies and infrastructure and hand hygiene technique.
\end{abstract}

Keywords: hand disinfection, preventive health services, hand hygiene, disease prevention.

\section{Resumo:}

Introdução: Embora a higiene das mãos seja a medida mais importante na mitigação do risco biológico em ambientes hospitalares, tem evidência científica que mostra baixo cumprimento das recomendações entre trabalhadores assistenciais. Objetivo: Avaliar

Notas de autor

a Autora de correspondencia. Correo electrónico: mercysal2003@yahoo.com 
o cumprimento de cinco dimensões que fortalecem a estratégia de higiene das mãos para a prevenção do risco biológico em ambientes assistenciais. Método: Estudo observacional, de corte transversal, em cinco instituições prestadoras de serviços de saúde. Foi aplicada estatística descritiva. Com o Teste da Mediana avaliou-se se havia diferenças significativas entre o grupo de profissionais por instituição de saúde, considerando significante uma $\mathrm{p} \leq 0,05$. Finalizou-se com análise discriminante. Resultados: A representatividade ocorreu nas instituições hospitalares de primeiro nível onde a equipe de enfermagem, seguido pelos médicos, apresentou melhor cumprimento do protocolo de lavagem de mãos. Esse porcentual foi impactado pelo menor cumprimento do fornecimento de suprimentos e infraestrutura. Há uma perfeita diferenciação entre os resultados do município de Cali em relação aos de Caicedonia, em contraste com os de Popayán, Buga e Buenaventura. Conclusão: Encontrou-se não inobservância do protocolo de higiene das mãos, com qualificação inaceitável para os fatores de suprimentos/infraestrutura e técnica de higiene das mãos.

Palavras-chave: desinfeção de mãos, serviços preventivos de saúde, higiene de mãos, prevenção de doença.

\section{Introducción}

La infección asociada a la atención médica (IAAM) representa un problema importante de seguridad y carga para los pacientes en los países en desarrollo, con un efecto grave y muy subestimado (1). Estas pueden aumentar la morbilidad y la mortalidad, crear resistencia antimicrobiana, y elevar la estancia hospitalaria lo cual redunda en costos de atención (2).

La Organización Mundial de la Salud (OMS) estima que cientos de millones de pacientes en todo el mundo son afectados anualmente por la IAAM. Por ejemplo, en Europa, más de 4 millones de pacientes se ven afectados al año y alrededor de 37.000 mueren por septicemias (3). Un estudio reciente en China identificó que el costo promedio de la hospitalización aumentó en $13.839,16$ yuanes $(1792,64)$ euros debido a la IAAM (4). En ese mismo sentido, en Estados Unidos se estima que 1,7 millones de las infecciones por año en hospitales están asociadas a la atención médica, resultando en aproximadamente 100.000 muertes con un costo de entre 20.000 y 40.000 millones de dólares (5). En contraste, países como Brasil, con un sistema nacional de información totalmente integral respecto a las IAAM no reporta estadísticas. Sin embargo, se estima que entre el $3 \%$ y $15 \%$ de los pacientes desarrolla algún tipo de infección (6).

La higiene de las manos se considera la medida con el mayor impacto y con el menor gasto para el control y la prevención de las infecciones derivadas de la atención médica, ya que evita la transmisión cruzada de microrganismos $(7,8)$, además de tener una asociación directa con la reducción de estas infecciones (9). Sin embargo, la falta de adherencia al protocolo de higiene de las manos en los trabajadores de la salud sigue siendo un problema a escala mundial (10). Las bajas tasas de cumplimiento varían entre el $5 \%$ y el $81 \%$, con un promedio del $40 \%(10,11)$. Son varias las razones expresadas para explicar el poco cumplimiento o no adherencia al protocolo de higiene de las manos, entre ellas: ausencia de capacitación en el tema, alergias a los jabones o alcohol glicerinado, falta de tiempo por excesiva carga de trabajo, problemas de accesibilidad a los insumos y puntos de higiene de las manos, falta de información científica que dé cuenta del impacto, falta de seguimiento al cumplimiento y participación activa de los trabajadores de la salud, deficiente de cultura de seguridad institucional y entrenamiento insuficiente (12).

Ante esta situación, la OMS, en el 2009, propuso una estrategia multimodal conformada por un conjunto de acciones orientadas a superar diferentes obstáculos y cambiar comportamientos riesgosos (13). La estrategia se basa en cinco componentes clave, relacionados con cambios institucionales, educación/ capacitación, evaluación y retroalimentación, recordatorios en el lugar de trabajo y un clima de seguridad institucional. En el contexto internacional, varios estudios han investigado su eficacia para mejorar cuantitativa y cualitativamente la adherencia al protocolo de higiene de las manos entre los profesionales de la salud (9,13); sin embargo, el tema aún es incipiente en Colombia. Algunos estudios publicados evalúan los conocimientos y las prácticas de higiene de las manos; pero ninguno ha evaluado la estrategia multimodal. En este contexto, el objetivo del presente estudio fue evaluar el cumplimiento de cinco componentes 
que fortalecen la estrategia de higiene de las manos para la prevención del riesgo biológico en ambientes asistenciales.

\section{Método}

Se realizó un estudio descriptivo de corte transversal en cinco instituciones prestadoras de servicios de salud (IPS) ubicadas en las ciudades de Cali, Caicedonia, Buga, Buenaventura y Popayán, en Colombia. Para la recolección de los datos se usó la lista de chequeo revisada, estandarizada y aplicada en el proyecto "Manos limpias atención segura”, estrategia multimodal para el mejoramiento de la higiene de las manos en Bogotá (14), la cual sigue el protocolo de autoevaluación del lavado de las manos de la OMS (15).

El instrumento consta de cuatro de los cinco componentes de la lista de chequeo para autoevaluación de la higiene de las manos de la OMS y 34 ítems. El primer componente (con 8 ítems) hace referencia a la disponibilidad de insumos e infraestructura para la higiene de las manos; el segundo (con 5 ítems), a los momentos de higiene de las manos; el tercero (con 14 ítems), a la técnica de higiene de las manos, y el cuarto (con 7 ítems), a la técnica de frote de manos con preparaciones alcohólicas.

La técnica de recolección de información fue la observación, hecha por maestrantes de un posgrado en Salud Ocupacional de una universidad privada de la ciudad de Cali, en 2017, después de revisar varios documentos sobre la higiene de manos de la OMS y de realizar prácticas por grupos en el aula de clase $(16,17)$. La calificación estuvo en función del cumplimiento o no de cada uno de los ítems sometidos a valoración. Dentro de las consideraciones éticas se tuvieron en cuenta los principios bioéticos de autonomía, justicia y beneficencia; se cataloga como un trabajo sin riesgo, y se tuvo en cuenta la aplicación del consentimiento informado para los participantes, así como los estándares de las normas científicas, técnicas y administrativas para la investigación en salud, de la Resolución 008430 de 1993 del Ministerio de Salud de la República de Colombia.

Para aplicar esta lista de chequeo se contó con información de las IPS, que solicitaron un informe ejecutivo con posibles estrategias de intervención, además de la evaluación del Comité de Investigaciones del programa académico del posgrado, el cual avaló la realización del trabajo. Se contactaron 93 profesionales, quienes en su totalidad aceptaron participar voluntariamente del proceso de observación de sus prácticas en el tema de higiene de las manos. Se recolectaron: 1) variables individuales relacionadas con la profesión, edad, cumplimiento de los momentos y técnica de higiene de las manos; así como la técnica de frotis de higiene de las manos en los trabajadores asistenciales. 2) Variables institucionales que consideraron nivel de complejidad de la IPS, el tipo de IPS, la disponibilidad de insumos y una infraestructura para la higiene de las manos antes de la atención, durante o después de esta.

Para el análisis se aplicó estadística descriptiva. Con el Test de Medianas se evaluó si había diferencias significativas entre el grupo de profesionales por institución de salud, considerando significante una $p \leq 0,05$. Se estableció el nivel en el cual se encontraban cada una de las instituciones de acuerdo con las prácticas de lavado de las manos y su promoción según la escala de calificación planteada en el proyecto "Manos limpias atención segura", llevado a cabo en el 2013 en Bogotá. Se finalizó con un análisis discriminante, con el cual se definieron los patrones diferenciadores de acuerdo con las prácticas de higiene de las manos y su promoción según ciudad.

\section{Resultados}

En el estudio se incluyeron 5 instituciones en las cuales se llevó a cabo el proceso de observación a 93 trabajadores asistenciales. El mayor porcentaje de encuestas fue aportado por la IPS ubicada en Cali, le 
siguieron en su orden Buga, Caicedonia, Popayán y Buenaventura. La tabla 1 presenta un resumen de las variables que caracterizaron a las instituciones y las profesionales que diligenciaron la encuesta.

TABLA 1.

Distribución de las frecuencias de las características generales de las IPS discriminado por rango edad de los trabajadores que diligenciaron la encuesta

\begin{tabular}{llll}
\hline Variable & Menores de 40 ańos & Mayores de 40 ańos & Edad del encuestado \\
\hline Ciudad & 29 & 8 & - \\
\hline Cali & 22 & 3 & - \\
\hline Buga & Sin dato & Sin dato & - \\
\hline Caicedonia & 8 & 5 & - \\
\hline Popayán & Sin dato & Sin dato & - \\
\hline Buenaventura & & & 7 \\
\hline Tipo de institución & 33 & 12 & - \\
\hline Hospital & 22 & 3 & 13 \\
\hline Homecare & 3 & - & 13 \\
\hline Centro médico/salud & & & 7 \\
\hline Nivel de complejidad & 37 & 8 & 20 \\
\hline Primer nivel & 6 & - \\
\hline Segundo y tercer nivel & 18 & & - \\
\hline Servicio & 23 & 4 & - \\
\hline Consulta externa & 15 & 3 & 3 \\
\hline Urgencias & 8 & 5 & 6 \\
\hline Hospitalización & 12 & 2 & 12 \\
\hline Atención domiciliaria & 12 & 5 & - \\
\hline Cargo & 16 & 6 & \\
\hline Médicos & 27 & 2 & \\
\hline $\begin{array}{l}\text { Enfermeras y auxiliares de } \\
\text { enfermería }\end{array}$ & 27 & 1 & \\
\hline $\begin{array}{l}\text { Otros profesionales } \\
\text { asistenciales }\end{array}$ & 11 & & \\
\hline Personal de apoyo & 4 & & \\
\hline
\end{tabular}

Fuente: elaboración propia

El análisis de cumplimiento global de los cuatro componentes de la lista de chequeo mostró una asimetría negativa en todos los resultados por debajo de la mediana y algunos valores extremos (encerrados en recuadro punteado en la parte inferior de la figura 1) de incumplimiento en el proceso de observación de la técnica y frote de higiene de las manos con preparaciones alcohólicas (figura 1).
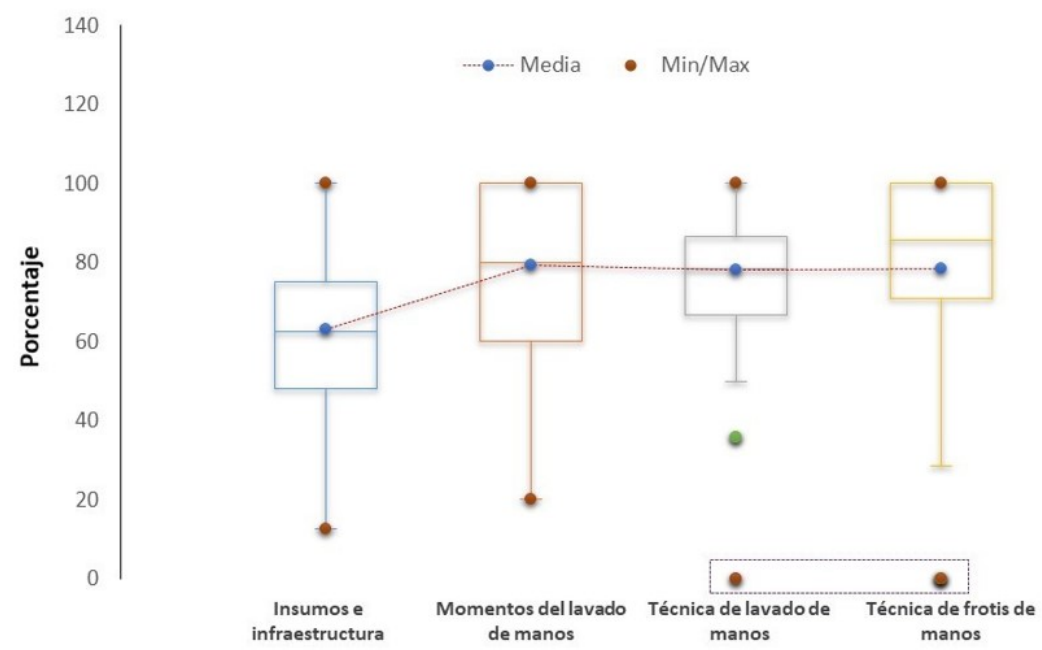

FIGURA 1.

Distribución de los subtotales de cumplimento de cada dimensión evaluada Fuente: elaboración propia

La tabla 2 presenta la calificación por componentes, discriminada por ciudad donde se ubicó la IPS fuente de información. Los resultados presentaron diferencias estadísticamente significativas en el cumplimiento de los cinco momentos de higiene de las manos entre los trabajadores asistenciales de la IPS ubicada en Cali, en comparación con los otros (test de medianas: $p=0,001$ ). De igual forma, el contraste de la puntuación del componente técnico de higiene de las manos por municipio evidenció que los participantes que residían en 
la ciudad de Cali obtuvieron mejores resultados que los de Buga (test de medianas: $p=0,025)$, Caicedonia (test de medianas, $p=0,001$ ) y Popayán (test de medianas, $p=0,000$ ), lo cual contrastó con Buenaventura (test de medianas: $p=1,000)$. Además, se observaron diferencias significativas entre el nivel de complejidad 1 en relación con el nivel 2 (test de medianas: $p=0,036$ ) y el nivel 3 (test de medianas: $p=0,000$ ).

TABLA 2.

Calificación de cada componente analizado discriminado por ciudad donde se ubicó la IPS fuente de información

\begin{tabular}{|c|c|c|c|c|c|c|c|c|c|c|c|c|c|c|c|c|}
\hline \multirow[t]{2}{*}{ Municipio } & \multicolumn{4}{|c|}{$\begin{array}{c}\text { Insumos e } \\
\text { infraestructura }\end{array}$} & \multicolumn{4}{|c|}{ Momentos de HM } & \multicolumn{4}{|c|}{ Técnica de HM } & \multicolumn{4}{|c|}{$\begin{array}{c}\text { Técnica de frote } \\
\text { de HM }\end{array}$} \\
\hline & O & A & D & I & O & A & D & I & $\mathbf{O}$ & A & D & I & O & A & D & I \\
\hline Cali & 36 & 1 & 0 & 0 & 4 & 8 & 0 & 25 & 9 & 6 & 7 & 15 & 2 & 2 & 5 & 24 \\
\hline Popayán & 3 & 0 & 6 & 2 & 4 & 4 & 0 & 3 & 6 & 4 & 0 & 1 & 4 & 5 & 1 & 1 \\
\hline Buga & 9 & 8 & 7 & 1 & 10 & 11 & 0 & 4 & 7 & 7 & 10 & 1 & 7 & 2 & 8 & 8 \\
\hline Caicedonia & 8 & 5 & 0 & 0 & 7 & 0 & 0 & 6 & 5 & 5 & 3 & 0 & 5 & 5 & 1 & 2 \\
\hline Buenaventura & 0 & 0 & 5 & 2 & 3 & 2 & 0 & 2 & 2 & 1 & 4 & 0 & 5 & 0 & 0 & 2 \\
\hline
\end{tabular}

O: óptimo, A: aceptable, D: deficiente, I: inaceptable; HM: higiene de las manos. Fuente: elaboración propia

El personal de enfermería fue el grupo de participantes que obtuvo las mejores puntuaciones en lo referente a la aplicación de los cinco momentos de higiene de las manos, técnica de higiene de las manos y frotis de manos con preparaciones alcohólicas; le siguen los médicos. En contraste, los profesionales del área de rehabilitación humana (fisioterapia y fonoaudiología) y personal de apoyo (conductores de ambulancias 1 y 2) tienen los puntajes más bajos, con diferencias estadísticamente significativas (test de medianas: $p=0,011)$ (figura 2).

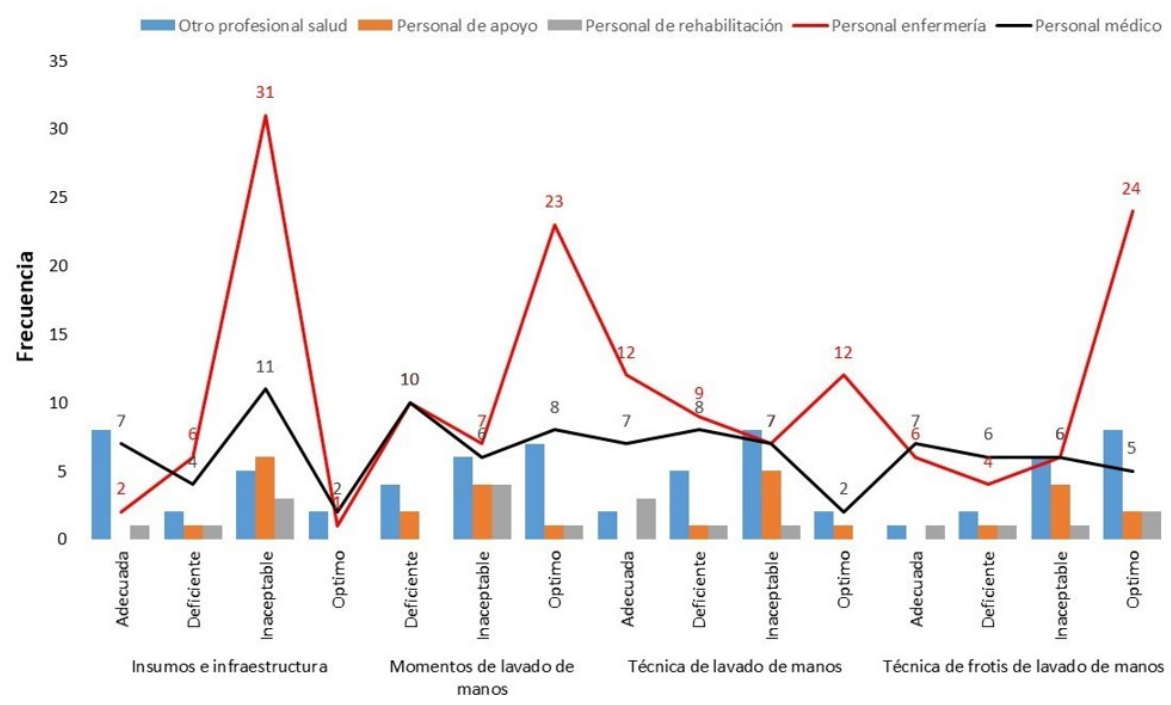

FIGURA 2.

Cumplimiento de las dimensiones que contribuyen al cumplimiento del protocolo de higiene de las manos por cargo Fuente: elaboración propia

Estos resultados mostraron una puntuación final por factor en la cual prevalece una calificación inaceptable para los factores de insumos e infraestructura $(n=56)$ y técnica de higiene de las manos $(\mathrm{n}=28)$. En la puntuación final, en los momentos de higiene de las manos y técnica de frotis de manos con preparaciones alcohólicas fue más frecuente el cumplimiento óptimo $(\mathrm{n}=40$ y $\mathrm{n}=41$, respectivamente) (tabla 3 ). 
TABLA 3.

Distribución de las calificaciones finales por factor de acuerdo con la puntuación final

\begin{tabular}{lll}
\hline Factor evaluado & Calificación & Porcentaje \\
\hline \multirow{4}{*}{ Insumos e infraestructura } & Inaceptable & 60,2 \\
\cline { 2 - 3 } & Deficiente & 15,1 \\
\cline { 2 - 3 } & Adecuada & 19,4 \\
\cline { 2 - 3 } & Óptimo & 5,4 \\
\hline \multirow{2}{*}{$\begin{array}{l}\text { Momentos de higiene de } \\
\text { las manos }\end{array}$} & Inaceptable & 29 \\
\cline { 2 - 3 } & Deficiente & 28 \\
\cline { 2 - 3 } & Óptimo & 43 \\
\hline \multirow{2}{*}{$\begin{array}{l}\text { Técnica de higiene de las } \\
\text { manos }\end{array}$} & Inaceptable & 30,1 \\
\cline { 2 - 3 } & Deficiente & 25,8 \\
\cline { 2 - 3 } & Adecuada & 25,8 \\
\cline { 2 - 3 } Técnica de frote de higien & Óptimo & 18,3 \\
\cline { 2 - 3 } de las manos & Deficiente & 24,7 \\
\cline { 2 - 3 } & Adecuada & 15,1 \\
\cline { 2 - 3 } & Óptimo & 44,1 \\
\hline
\end{tabular}

Fuente: elaboración propia

Finalmente, un análisis discriminante muestra dos ejes factoriales, los cuales explican el 95,36\% de las diferencias. La figura 3 presenta las observaciones sobre los dos ejes factoriales seleccionadas. La distribución de los datos da cuenta de una dispersión muy amplia con relación al cumplimiento de cada componente en los municipios de Popayán y Buenaventura, los cuales corresponde al menor número de listas de chequeo aplicadas ( 11 y 7 , respectivamente). Así, se graficaron los centroides de cada municipio, con lo cual se visualiza que las observaciones están correctamente discriminadas en los municipios de Cali y Caicedonia sobre los ejes factoriales obtenidos a partir de las variables explicativas iniciales; sin embargo, los resultados de los municipios de Popayán, Buga y Buenaventura no (figura 3).
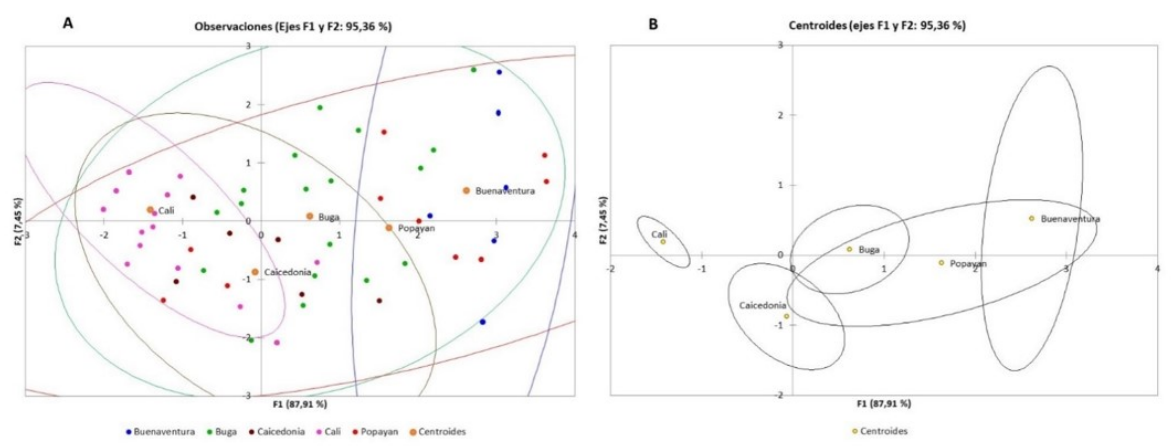

FIGURA 3.

Distribución de las observaciones (A) y centroides (B) de los datos en los planos factoriales discriminantes Fuente: elaboración propia

\section{Discusión}

Después de más de 60 años de demostrar la necesidad de la higiene de las manos como una medida preventiva frente a la aparición de infecciones y la mitigación del riesgo biológico en trabajadores asistenciales, continúa una baja adherencia de parte del personal sanitario en el ámbito hospitalario (18-20), situación que también se evidencia en los resultados de la presente investigación, cuando al analizar el cumplimiento global de los cuatro componentes de la lista de chequeo, se halló una asimetría negativa en todos los resultados. Sin embargo, la calificación fue mejor en la ciudad de Cali, donde el personal de enfermería, seguido por el médico, fueron los mejores evaluados en los cinco componentes. 
El cumplimiento de protocolos de higiene de manos difiere en los reportes de diferentes investigaciones internacionales: ellos pueden variar del $33 \%$ al $65 \%(18,19)$. En este estudio, los momentos y la técnica de frote fueron óptimos en una proporción muy alta; sin embargo, la técnica de higiene de las manos fue deficiente e inaceptable en más del $50 \%$ de las listas de chequeo. Estos resultados contrastaron con los de Musu et al. (8), quienes evidenciaron que en los cinco momentos de higiene de las manos el personal médico (médicos y personal de enfermería) mostró un rango de cumplimiento del $28 \%$ y del $86 \%$, respectivamente. En términos generales, ellos tienen las mejores prácticas en higiene de manos, tal y como lo indican Pitter y Allegranzi (20), para quienes la tasa de cumplimiento de los trabajadores asistenciales (médicos y enfermeras) fue del 38,7 \% en ausencia de intervenciones específicas (12). En otro estudio, Rynga et al. (21) mostraron que la mayoría de los trabajadores asistenciales informaron que conocían los momentos y la técnica de higiene de las manos ( $89,3 \%$ y $96 \%$, respectivamente); sin embargo, solo el 47,9 \% conocía el tiempo necesario para el frote con alcohol en las manos.

Entre los médicos encuestados, la razón más común informada para no practicar una correcta higiene de las manos fue la falta de accesibilidad de los lavamanos o el desinfectante a base de alcohol en el punto de atención. La infraestructura necesaria de fácil acceso y funcionamiento óptimo es indispensable. Los trabajadores que puntuaron con la calificación más baja en este estudio involucraron o los de rehabilitación humana y conductores. Estos resultados contrastaron con los de Yin et al. (22), quienes indicaron que para unas actividades asistenciales el personal médico tiene mejor cumplimiento (70\%), seguido por los fisioterapeutas (62\%), enfermeras (49\%) y tecnólogas en enfermería (48\%); entre tanto, en otros trabajos el personal de enfermería tuvo mejor cumplimiento del protocolo de higiene de las manos.

Rynga et al. (21) refirieron que en su estudio, en muchas áreas hospitalarias, los lavamanos no eran fácilmente accesibles y no había facilidad para secarse las manos después del lavado. Frotaciones de manos a base de alcohol estaban presentes, pero no fácilmente por los costos. Los carteles que ilustran las técnicas de higiene de las manos estaban presentes solo en algunos lugares, y en los servicios donde se ubicaron los recordatorios, la calificación final fue superior.

En resumen, el cumplimiento de los componentes para una buena higiene de manos desborda los aspectos relacionados con el comportamiento de los trabajadores sanitarios y se ubica en la esfera de la infraestructura, la disponibilidad de recursos económicos y la gestión. Para llevar a cabo la higiene de las manos es importante la disponibilidad de los productos o de las preparaciones de alcohol glicerinado $(23,24)$. Entre las principales barreras para su implementación están el déficit de infraestructura — por ejemplo, falta de agua, jabón o desinfectantes para manos; lavamanos bloqueados o con fugas, e instalaciones mal ubicadas- (25). El hecho de que las instituciones de segundo y tercer nivel de complejidad tuvieran mejores puntuaciones, en relación con las del primer nivel, es una evidencia de los mayores recursos disponibles para los programas de atención segura y mitigación de riesgos dirigidos a los profesionales asistenciales y a los pacientes. En este sentido, Ibeneme et al. (26) y Ataiyero et al. (24) coinciden en que el cumplimiento del protocolo de higiene de las manos difiere entre las instituciones hospitalarias según su fuente de financiación (pública o privada), así como según el nivel de complejidad.

Esta investigación usó la técnica de observación como elemento para recolectar información, la cual para algunos autores puede generar el efecto Hawthorne, en el cual se modifica el cumplimiento dependiendo de quién observe: si es encubierto o personal hospitalario encargado del control de infecciones $(8,27)$. Cuando en la evaluación participan observadores reconocidos, los resultados se asocian con tasas más altas de cumplimiento de la higiene de las manos (28), incluso se establecen recomendaciones en el tiempo de observación para controlar este efecto, a periodos de aproximadamente 15 minutos (8). Para esta investigación, las personas que llevaron a cabo la verificación de la lista de chequeo no eran observadores reconocidos, y aunque se solicitó el consentimiento informado, nunca se informó al personal asistencial a partir de qué fecha se haría; por tanto, los resultados se ajustan más a la realidad. 
Debido a los inconvenientes que puede traer la observación directa, se están usando otros dispositivos como: contadores electrónicos de higiene de las manos (29), sensores que detectan vapores de alcohol (30) o radiofrecuencia para determinar si ha ocurrido un procedimiento de higiene de las manos (31); cámaras de video con vistas de cada lavamanos (32); insignias de radiofrecuencia (33); entre otras. Las tecnologías buscan medir y mejorar el cumplimiento, y son el futuro para la higiene de manos; sin embargo, los costos pueden retrasar su implementación (29).

Aun cuando en el ambiente asistencial no se puede lograr un "riesgo cero" tanto para el trabajador de la salud como para el paciente, se puede administrar de manera eficaz, eliminando las causas raíz de la no adherencia. Los resultados de esta investigación sugieren que si bien la higiene de las manos es la línea de acción principal para reducir el riesgo de infecciones, su importancia aún no es bien reconocida. La evidencia sugiere que una buena manera de aumentar el cumplimiento de este protocolo es proporcionar mejoras continuas en la calidad de los protocolos y procedimientos, y apoyarlos en términos de suministro de insumos, comunicación, educación y capacitación $(14,16,17,19)$.

\section{Conclusiones}

Los resultados señalan la necesidad para las instituciones y para su personal asistencial de ajustarse al cumplimiento del protocolo de higiene de las manos. Sin embargo, este compromiso debe ir acompañado del suministro de insumos e infraestructura, en los diferentes servicios que coadyuven al cumplimiento del protocolo, así como la sensibilización y capacitación en la estrategia multidimensional para todo el personal asistencial.

\section{Referencias}

1. Li Shen, An J, An J, et al. Implementation of WHO multimodal strategy for improvement of hand hygiene: a quasi-experimental study in a Traditional Chinese Medicine hospital in Xi'an, China. Antimicrob Resist Infect Control. 2017;6:98. https://doi.org/10.1186/s13756-017-0254-4

2. Pereira EBS, Jorge MT, Oliveira EJ, Júnior ALR, Santos LRL, Mendes-Rodrigues C. Evaluation of the multimodal strategy for improvement of hand hygiene as proposed by the World Health Organization. J Nurs Care Qual. 2017;32(2):E11-9. https://doi.org/10.1097/ncq.000000000000019

3. World Health Organization. Report on the burden of endemic health care-associated infection worldwide [internet]. Geneva, Switzerland: WHO/Patient Safety; 2011. Disponible en: https://apps.who.int/iris/bitstream/10665/ 80135/1/9789241501507eng.pdf

4. Jia H, Hou T, Li W, Ma Q, Liu W, Yang Y, et al. Economic loss due to healthcare-associated infection in 68 general hospitals in China. Chinese J Infect Control. 2016;15:637-41. https://doi.org/10.1155/2019/2590563

5. Zimlichman E, Henderson D, Tamir O, et al. Health care-associated infections: a meta-analysis of costs and financial impact on the US health care system. JAMA Intern Med. 2013;173(22):2039-46. https://doi.org/10.1001/jam ainternmed.2013.9763

6. Junior CN, Mello DS, Padoveze MC, et al. Characterization of epidemiological surveillance systems for healthcareassociated infections (HAI) in the world and challenges for Brazil. Cad Saude Publica. 2014;30(1):11-20. http s://doi.org/10.1590/0102-311X00044113

7. Chen JK, Wu KS, Lee SS, et al. Impact of implementation of the World Health Organization multimodal hand hygiene improvement strategy in a teaching hospital in Taiwan. Am J Infect Control. 2016;44:222-7. https:// doi.org/10.1016/j.ajic.2015.10.004

8. Musu M, Lai A, Mereu NM, et al. Assessing hand hygiene compliance among healthcare workers in six Intensive Care Units. J Prev Med Hyg. 2017;58(3):E231-7. 
9. Valim MD, Rocha ILS, Souza TPM, Cruz YAD, Bezerra TB, Baggio É, Morais RB, Ribeiro AC. Efficacy of the multimodal strategy for hand hygiene compliance: an integrative review. Rev Bras Enferm. 2019 Apr;72(2):552-65. http://dx.doi.org/10.1590/0034-7167-2018-0584

10. Alshehari AA, Park S, Rashid H. Strategies to improve hand hygiene compliance among healthcare workers in adult intensive care units: a mini systematic review. J Hosp Infect. 2018;100(2):152-8. https://doi.org/10.1016/j.jhi n.2018.03.01

11. Squeri R, Genovese C, Palamara MAR, Trimarchi G, La Faucil V. "Clean care is safer care": correct handwashing in the prevention of healthcare associated infections. Ann Ig. 2016;28:409-15. https://doi.org/10.7416/ai.201 6.2123

12. Teker B, Ogutlu A, Gozdas HT, Ruayercan S, Hacialioglu G, Karabay O. Factors affecting hand hygiene adherence at a private hospital in Turkey. Eurasian J Med. 2015;47(3):208-12. https://doi.org/10.5152/eurasianjmed.20 15.78

13. Oliveira AC, Gama CS, Paula AO. Multimodal strategy to improve the adherence to hand hygiene and selfassessment of the institution for the promotion and practice of hand hygiene.J Public Health. 2018;40(1):163-8. https://doi.org/10.1093/pubmed/fdx035

14. Secretaría Distrital de Salud, Asociación Colombiana de Infectología Capítulo Central. Protocolo de lavado de manos limpias atención segura: estrategia multimodal para el mejoramiento de la higiene de lavado de protocolo de lavado de manos en Bogotá [internet]. Bogotá. Disponible en: http://www.saludcapital.gov.co/sitios/Vigila nciaSaludPublica/Todo\%20IIH/Estrategia\%20Distrital\%20Manos\%20Limpias\%20Atencion\%20Segura.pdf

15. Organización Mundial de la Salud. Marco de autoevaluación de la higiene de las protocolo de lavado de manos 2010: Introducción e instrucciones de uso [internet]. Disponible en: https://www.who.int/gpsc/country_wor $\mathrm{k} / \mathrm{hhsa} \_$framework_es.pdf?ua $=1$

16. Organización Mundial de la Salud. Directrices sobre componentes básicos para los programas de prevención y control de infecciones a nivel nacional y de establecimientos de atención de salud para pacientes agudos [internet]. Washington, D. C.: Organización Panamericana de la Salud; 2016. Disponible en: https://apps.who.int/iris/bitstream/handle/10665/255764/9789275319635-spa.pdf;jsessionid=2B08B6 4456F567460094B9B77B93F660? sequence=1

17. Organización Mundial de la Salud. Higiene de las protocolo de lavado de manos en la atención sanitaria ambulatoria/residencias de la tercera edad y a domicilio [internet]. Disponible en: https://www.who.int/gpsc/ 5may/Hand_Hygiene_When_How_Leaflet_OPC_Sp_web_2012.pdf?ua $=1$

18. Schwadtke L, Graf K, Lutze B, von Lengerke T, Chaberny IF. Compliance with hand hygiene guidelines on intensive care units at a university medical centre with surgical focus. Deutsche Medizinische Wochenschrift. 2014;139(25-26):1341-5. https://doi.org/10.1055/s-0034-137014

19. Karaaslan A, Kepenekli Kadayifci E, Atıcı S et al. Cumplimiento de los trabajadores de la salud con las prácticas de higiene de protocolo de lavado de manos en unidades de cuidados intensivos neonatales y pediátricos: observación abierta: perspectiva interdisciplinaria. Infect Dis. 2014;2014:306478. https://doi.org/10.1155/2014/306478

20. Pitter D, Allegranzi B. Hand hygiene promotion strategies. Inhand hygiene: a handbook for medical professionals. Florida: Wiley-Blackwell; 2017. p. 123-33.

21. Rynga D, Kumar S, Gaind R, Kumar Rai A. Hand hygiene compliance and associated factors among health care workers in a tertiary care hospital: self-reported behaviour and direct observation. Int J Infect Control. 2017;13(1). https://doi.org/10.3396/ijic.v13i1.17137

22. Yin J, Reisinger HS, Vander Weg M, Schweizer ML, Jesson A, Morgan DJ, Forrest G, Graham M, Pineles L, Perencevich. Establishing evidence-based criteria for directly observed hand hygiene compliance monitoring programs: a prospective, multicenter cohort study. Infect Control Hosp Epidemiol. 2014 Sep;35(9):1163-8. h ttps://doi.org/10.1086/677629

23. Sopjani I. Health care personnel's attitude toward hand hygiene in regard to the prevention of health-care associated infections: a cross sectional study at the University Hospital Pristine. Open Journal of Nursing. 2016;6:841-52. https://doi.org/10.4236/ojn.2016.610083 
24. Ataiyero Y, Dyson J, Graham M. Barriers to hand hygiene practices among health care workers in sub-Saharan African countries: a narrative review. Am J Infect Control. 2018;47(5):565-73. https://doi.org/10.1016/j.ajic. 2018.09.014

25. Dreidi MM, Alrimawi I, Saifan AR, Batiha A-M. Hand hygiene knowledge, practices and attitudes among nurses and physicians. Health. 2016;8:456-62. https://doi.org/10.4236/health.2016.85048

26. Ibeneme S, Maduako V, Ibeneme GC, et al. Hand hygiene practices and microbial investigation of hand contact swab among physiotherapists in an Ebola endemic region: implications for public health. Biomed Res Int. 2017;5841805:1-14.

27. Werzen A, Thom KA, Robinson GL, et al. Comparing brief, covert, directly observed hand hygiene compliance monitoring to standard methods: a multicenter cohort study. Am J Infect Control. 2019;47(3):346-8. https:// doi.org/10.1016/j.ajic.2018.08.015

28. Kurtz S. Measuring and accounting for the Hawthorne effect during a direct overt observational study of intensive care unit nurses. Am J Infect Control. 2017;45(9):995-1000.

29. Albright J, White B, Pedersen D, Carlson P, Yost L, Littau Ch. Use patterns and frequency of hand hygiene in healthcare facilities: analysis of electronic surveillance data. Am J Infect Control. 2018;46(10):1104-109.

30. Alshehari A, Park S, Rashid H. Strategies to improve hand hygiene compliance among health care workers in adult intensive care units: a mini systematic review. J Hosp Infect. 2018;100(2):152-5.

31. Ribeiro de Macedo RD, Oliveira EM, Pio da Silva, V et al. Positive deviance: using a nurse call system to evaluate hand hygiene practices. Am J Infect Control. 2012;40:946-50.

32. Marraa AR, Edmondb MB. New technologies to monitor healthcare worker hand higiene. Clin Microbiol Infect. 2014;20(1):29-33. https://doi.org/10.1111/1469-0691.12458

33. Pineles L, Morgan DJ, Limper HM, Weber SG, Thom KA, Perencevich EN, Harris A D, Landon EM. Accuracy of a radiofrequency identification (RFID) badge system to monitor hand hygiene behavior during routine clinical activities. Am J Infect Control. 2014; 42(2):144-7. https://doi.org/10.1016/j.ajic.2013.07.014

\section{Notas}

* Artículo original de investigación

\section{Licencia Creative Commons CC BY 4.0}

Cómo citar este artículo: Salcedo-Cifuentes M, Ordóñez-Hernández CA, Calvo-Soto AP. Cumplimiento de una estrategia de higiene de las manos en ambientes asistenciales. Investig Enferm Imagen Desarr. 2020;22. https://doi.org/10.11144/Javeriana.ie22.cehm 